\title{
The Stability Analysis of the Strategic Transaction in a Deregulation Environment
}

\author{
Chong-Yih Li Chih-Wen Liu \\ Department of Electrical Engineering \\ National Taiwan University \\ Taipei, Taiwan
}

\begin{abstract}
:
In a deregulation system, the transaction may happen at any participant of the power pool. That will result in the complexity of the power exchange. The supervisor is necessary to inspect the system stability. In the paper, we derive the dynamic equations for the three distinct deregulation policies, uniform policy, UK (United Kingdom) policy, and discriminatory policy. The dynamic equation can deal with the strategic bidding and permit the distinct buying price. According to the simulation results, it is obvious that the unstable priority is uniform, UK, and discriminatory policy. Beside that, we also portray the unstable region in the power exchange region. They are useful for congestion management of ISO (Independent Power Operator).
\end{abstract}

Keyword: ISO, power pool, deregulation, SMP, clearing price.

\section{I . Introduction}

In a deregulation system, each participant tries to acquire the most benefit through the spot power market. It is very different from the conventional economic dispatch that the object is the entire minimum operation cost. In the deregulation system, power company may lose some member's profit to make transmission line congested. Moreover, its other member will bid the more expensive price to compensate the deficient power and so more company profits are obtained. This is called congestion benefit. Therefore, the strategic behavior of congestion benefit make the system management more complex. It is necessary to examine the stability of strategic transaction.

Some papers use the game theory [1] to study the strategic benefits. The papers $[2,3]$ discussed that the network imposed additional constraints on the bids, and provider will increase their benefits by using game theory to coordinate bidding strategy. A genetic algorithm is used to evolve the bidding strategies and predicts whether the strategies will be profitable [4]. The strategic bidding is also discussed in a brokerage system [5-8]. The tariff can be used for the reference of strategic behavior [9-11].
Some authors [12-13] use the differential equation to study the market stability. In paper [14], the author establishes the differential algebraic equation (DAE), and examines the stability by analyzing the eigenvalues of its characteristic equation. It is successful to analyze the uniform price and non-strategic system. However, it is hard to analyze the actual power system that strategic behavior and the distinct prices for each transaction often exists. Our purpose is to obtain the dynamic equations that realizes the stability analysis for three distinct deregulation policies, uniform policy, U.K. policy and discriminatory policy.

There are four sections in the paper. In section II, the dynamic equations are derived for the distinct policies to analyze its stability of the steady solution. In section III, we applied our proposed differential equations to IEEE 30-bus [15]. The stability between the policies is discussed. A quantitative description of unstable region supports ISO for congestion management. Finally, a brief conclusion is outlined in section IV.

\section{The Stability Analysis of the Distinct Deregulation Policies}

To study the subject of the stability, we give the 4-bus example in Fig. 1 .

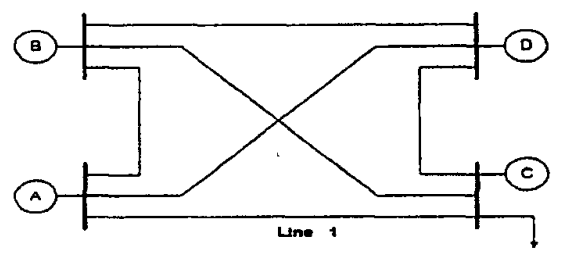

Fig. 1 : The configuration of 4-bus system

The terminology in the paper:

$P_{i}$ : Generation by unit $i$, in $M W$.

$\mathrm{L}$ : Total load, in $\mathrm{MW}$

$D$ : Total delivery power of $P_{A}, P_{B}$ and $P_{C}$

$E_{1}$ : Capacity of line 1

$\alpha$ : Fraction of A's generation flowing on line 1

$\beta$ : Fraction of B's generation flowing on line 1

$S^{\mathrm{MAX}}$ : Maximum price allowed by pool in $\$ / \mathrm{MW}$

$S_{i}$ : Unit cost of generator $i$, in $M W$ 
$\mathrm{P}_{\mathrm{i}}^{*}$ : Pre-curtailment schedules for generator $\mathrm{i}$, in $\mathrm{MW}$

$\lambda$ : Pool buying price

$b_{i}$ : The first order coefficient of generator $i$ marginal cost

$C_{i}$ : The second order coefficient of generator i marginal

cost

$T_{i}$ : The unit reacting time constant in generator $i$.

$G_{i}$ : Provider $i$, for $i=A, B, C, D$

$f_{i}$ :The profit of company $i$

Assume transaction procedure is distinguished into two steps by Line 1 congestion. The interesting providers are $G_{A}$ and $G_{B}$ prior the Line 1 congestion. If the line 1 congestion occurs, the pool will curtail some original transaction power and search another provider to compensate the deficient amount. Let the $G_{C}$ and $G_{D}$ to fill the deficient amount. Suppose $G_{A}$ and $G_{C}$ belongs to the same company 1 . The $G_{B}$ and $G_{D}$ belongs to company 2 and company 3 , respectively. When $G_{A}$ and $G_{C}$ use strategy to get congestion benefit, the stability of the distinct policies, described at [16], is studied here.

We will derive the optimal dynamic equation of the different deregulation policies first. Moreover, we append the strategic function into the optimal dynamic equation to make the stability analysis available for the strategic behavior. For all of the policies, we assume the bidding price of $G_{B}$ is greater than the $G_{A}$ 's and the Line 1 is more sensitive for $G_{A}$ than $G_{B}$ (it is $\alpha>\beta$ ). In addition, the buying prices of $G_{C}$ and $G_{D}$ are $S^{M A x}$. There is no reason for them to bid their price lower the $S^{m A x}$, because that the pool certainly needs their power to maintain power balance. These dynamic equations are derived as the following.

\section{Uniform policy}

The buying prices of each provider are the same under the uniform policy. Here, we set $\lambda$ to denote the buying price. If we neglect the constant term of unit marginal cost, the company profit is expressed as the following:

$$
\begin{aligned}
& f_{1}\left(P_{A}, P_{C}\right)=\left(\lambda-b_{A}\right) P_{A}-0.5 C_{A} P_{A}{ }^{2}+\left(\lambda-b_{c}\right) P_{c}-0.5 C_{c} P_{c}{ }^{2}(1) \\
& f_{2}\left(P_{B}\right)=\left(\lambda-b_{B}\right) P_{B}-0.5 C_{B} P_{B}{ }^{2} \\
& f_{3}\left(P_{D}\right)=\left(\lambda-b_{D}\right) P_{D}-0.5 C_{D} P_{D}{ }^{2}
\end{aligned}
$$

where $f_{1}, f_{2}, f_{3}$ are the company profits for company $1,2,3$, respectively. The strategic behavior is explained as the $P_{A}$ uses the line congestion constraint to make $P_{C}$ generate. The more $P_{C}$ amount is expected to obtain more benefits of company 1 . The $P_{C}$ can be expressed as the following:

$$
P_{C}=D-P_{A}-P_{B}=\left(D-E_{1} / \beta\right)+A P_{A}
$$

Where $A=-1+\alpha / \beta$ and $D$ is total delivery power of $P_{A}, P_{B}$, and $\mathrm{P}_{\mathrm{C}}$.

According to Eq.(4), we have the following equation.

$$
\partial P_{C} / \partial P_{A}=A
$$

The constraint equation is the following:

$$
\mathrm{P}_{\mathrm{A}}+\mathrm{P}_{\mathrm{B}}+\mathrm{P}_{\mathrm{C}}+\mathrm{P}_{\mathrm{D}}=\mathrm{L}
$$

According to the reference [14], we can express the dynamic and constraint equations as the following compact form:

$$
\left[\begin{array}{lllll}
T_{A} & 0 & 0 & 0 & 0 \\
0 & T_{B} & 0 & 0 & 0 \\
0 & 1 & T_{C} & 0 & 0 \\
0 & 0 & 0 & T_{D} & 0 \\
0 & 0 & 0 & 0 & 0
\end{array}\right]\left[\begin{array}{l}
P_{A} \\
P_{B} \\
P_{C} \\
P_{D} \\
\lambda
\end{array}\right]=Q\left[\begin{array}{l}
P_{A} \\
P_{B} \\
P_{C} \\
P_{D} \\
\lambda
\end{array}\right]+B
$$

$$
O=\left[\begin{array}{cccccc}
-\left(C_{A}+A^{2} C_{C}\right) & 0 & 0 & 0 & 1+A \\
0 & -C_{B} & 0 & 0 & 1 \\
0 & 0 & -\left(C_{C}+C_{A} / A^{2},\right. & 0 & 1+1 / A \\
0 & 0 & 0 & -C_{D} & 1 \\
1 & 1 & 1 & 1 & 0
\end{array}\right]
$$

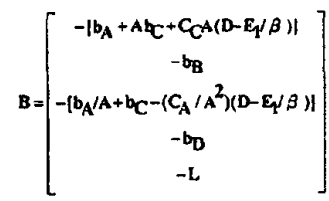

\section{U.K policy with Merit-Order Curtailment}

The power pool buying prices are two levels due to line congestion in the UK policy. We set two distinct buying prices to denote it, $\lambda_{1}$ for $G_{A}, G_{B}$ and $\lambda_{2}$ for $G_{C}, G_{D}$. To avoid the congestion, we will curtail the $G_{B}$ power.

The matrix form of dynamic and constraint equations are derived as follows:

$$
\left[\begin{array}{cccccc}
T_{A} & 0 & 0 & 0 & 0 & 0 \\
0 & T_{D} & 0 & 0 & 0 & 0 \\
0 & 0 & T_{B} & 1 & 0 & 0 \\
0 & 0 & 0 & T_{C} & 0 & 0 \\
0 & 0 & 0 & 0 & 0 & 0 \\
0 & 0 & 0 & 0 & 0 & 0
\end{array}\right]\left[\begin{array}{l}
P_{A} \\
P_{D} \\
P_{B} \\
P_{C} \\
\lambda_{1} \\
\lambda_{2}
\end{array}\right]=Q\left[\begin{array}{l}
P_{A} \\
P_{D} \\
P_{B} \\
P_{C} \\
\lambda_{1} \\
\lambda_{3}
\end{array}\right]+B
$$

$$
\begin{aligned}
& Q=\left[\begin{array}{llllll}
-\left(C_{A}+A^{2} C_{C}\right) & 0 & 0 & 0 & 1 & A \\
0 & -C_{D} & 0 & 0 & 0 & 1 \\
0 & 0 & -C_{B} & 0 & 1 /(1-A) & 0 \\
0 & 0 & 0 & -\left(C_{C}+C_{A} / A^{2}\right) & 1 / A & 1 \\
1 & 0 & 1 & 0 & 0 & 0 \\
0 & 1 & 0 & 1 & 0 & 0
\end{array}\right] \\
& B=\left[\begin{array}{c}
-\left(b_{A}+A b_{C}+C_{C} A\left(D-E_{t^{\prime}} / B\right)\right] \\
-b_{D} \\
-b_{B} \\
-\left(b_{A} / A+b_{C}-\left(C_{A} / A^{2}\right)\left(D-E_{t}(B)\right)\right] \\
-K \\
-(L-K)
\end{array}\right]
\end{aligned}
$$


Here we have arranged power vector in the sequence of $P_{A}, P_{D}, P_{B}, P_{C}$ to prevent singularity when we compute the eigenvalues of the system later. The symbol $K$ is the sum of $P_{A}$ and $P_{B}$.

\section{U.K policy with least curtailment:}

The pool buying prices are still two levels due to congestion. To avoid the congestion, we have to curtail $G_{A}$.

The compensation payment is paid to $G_{A}$.

The matrix form of dynamic and constraint equations are derived as follows:

$$
\begin{aligned}
& {\left[\begin{array}{llllll}
T_{A} & 0 & 0 & 0 & 0 & 0 \\
0 & T_{D} & 0 & 0 & 0 & 0 \\
0 & 0 & T_{B} & 0 & 0 & 0 \\
0 & 0 & 0 & T_{C} & 0 & 0 \\
0 & 0 & 0 & 0 & 0 & 0 \\
0 & 0 & 0 & 0 & 0 & 0
\end{array}\right]\left[\begin{array}{l}
\dot{P}_{A} \\
\dot{P}_{D} \\
\dot{P}_{B} \\
\dot{P}_{C} \\
i_{1} \\
\dot{\lambda}_{2}
\end{array}\right]=0\left[\begin{array}{l}
P_{A} \\
P_{D} \\
P_{B} \\
P_{C} \\
i_{1} \\
\lambda_{2}
\end{array}\right]+B} \\
& Q=\left[\begin{array}{cccccc}
-\left(-C_{A}+A^{2} C_{C}\right) & 0 & 0 & 0 & 1+A & A \\
0 & -C_{D} & 0 & 0 & 0 & 1 \\
0 & 0 & -C_{B} & 0 & 1 & 0 \\
0 & 0 & 0 & -\left\langle C_{C}+C_{A} / A^{2}\right) & 1+1 / A & 1 \\
1 & 0 & 1 & 0 & 0 & 0 \\
0 & 1 & 0 & 1 & 0 & 0
\end{array}\right] \\
& B=\left[\begin{array}{c}
\left.-\mid b_{A}+A b_{C}+C_{C^{A}} A\left(D-E_{1} / \beta\right)\right] \\
-b_{D} \\
-b_{B} \\
\left.-1 b_{A} / A+b_{C}-\left(C_{A} / A^{2}\right)\left(D-E_{1} / \beta\right)\right) \\
-K \\
-(L \cdot K)
\end{array}\right]
\end{aligned}
$$

\section{Discriminatory policy with merit-order curtailment:}

The pool buying prices are according to the provider's biding price and no curtailment compensation is needed to pay. Before congestion, set the bidding prices of $P_{A}$ and $P_{B}$ to be $\lambda_{1}, \lambda_{2}$ with that $\lambda_{1}$ is lower than $\lambda_{2}$. After the congestion, it is intuitive that the $P_{C}$ and $P_{D}$ will bid the maximum allowable buying price $S^{\mathrm{MAX}}$. So we set the same price to be $\lambda_{3}$.

The matrix form of dynamic and constraint equations are derived as follows:

$$
\left[\begin{array}{ccccccc}
T_{A} & 0 & 0 & 0 & 0 & 0 & 0 \\
0 & T_{D} & 0 & 0 & 0 & 0 & 0 \\
0 & 0 & T_{B} & 0 & 0 & 0 & 0 \\
0 & 0 & 0 & T_{C} & 0 & 0 & 0 \\
0 & 0 & 0 & 0 & 0 & 0 & 0 \\
0 & 0 & 0 & 0 & 0 & 0 & 0 \\
0 & 0 & 0 & 0 & 0 & 0 & 0
\end{array}\right]\left[\begin{array}{l}
\dot{P}_{A} \\
\dot{P}_{D} \\
\dot{P}_{B} \\
\dot{P}_{C} \\
\lambda_{1} \\
\lambda_{2} \\
\lambda_{3}
\end{array}\right]=Q\left[\begin{array}{c}
P_{A} \\
P_{D} \\
P_{B} \\
P_{C} \\
\lambda_{1} \\
\lambda_{2} \\
\lambda_{3}
\end{array}\right]+B
$$

$$
Q=\left[\begin{array}{ccccccc}
-\left(C_{A}+A^{2} C_{C}\right) & 0 & 0 & 0 & 1 & 0 & A \\
0 & -C_{D} & 0 & 0 & 0 & 0 & 1 \\
0 & 0 & -C_{B} & 0 & 0 & 1 & 0 \\
0 & 0 & 0 & -\left(C_{C}+C / A^{2}\right) & 1 / A & 0 & 1 \\
1 & 0 & 0 & 0 & 0 & 0 & 0 \\
0 & 0 & 1 & 0 & 0 & 0 & 0 \\
0 & 1 & 0 & 1 & 0 & 0 & 0
\end{array}\right]
$$

$$
B=\left[\begin{array}{c}
\left.-\mid b_{A}+A b_{C}+C_{C} A\left(D-E_{1} / A\right)\right] \\
-b_{D} \\
-b_{B} \\
-\left[b_{A} / A+b_{C}-\left(C_{A} / A^{2}\right)\left(D-E_{1} / B\right)\right] \\
-K 1 \\
-K 2 \\
-(L-K L-K 2)
\end{array}\right]
$$

The $K_{1}$ is the transaction amount of $P_{A}$. The $K_{2}$ is the transaction amount of $\mathrm{P}_{\mathrm{B}}$. Assign the three different transaction amounts $K_{1}, K_{2}$ and $L-K_{1}-K_{2}$ corresporid to the three different prices $\lambda_{1}, \lambda_{2}$ and $\lambda_{3}$, respectively.

\section{Discriminatory policy with least curtailment:}

For the merit order curtailment, the $G_{B}$ power is curtailed and the deficient power is compensated by $\left(P_{C}+P_{D}\right)^{\text {merit-order }}$. For the least curtailment, the $G_{A}$ power is curtailed and the deficient power is compensated by $\left(\mathrm{P}_{\mathrm{C}}+\mathrm{P}_{\mathrm{D}}\right)^{\text {ieast }}$. Then, the two power compensations have the following relation.

$$
\left(\mathrm{P}_{\mathrm{C}}+\mathrm{P}_{\mathrm{D}}\right)^{\text {least }}=(\beta / \alpha)\left(\mathrm{P}_{\mathrm{C}}+\mathrm{P}_{\mathrm{D}}\right)^{\text {merit-order }}
$$

\section{The Stability Analysis:}

The general form of the above matrix of dynamic and constraint equations can be written as the following form

$$
\left[\begin{array}{ccc}
\mathrm{T}_{1} & 0 & 0 \\
0 & \mathrm{~T}_{2} & 0 \\
0 & 0 & 0
\end{array}\right]\left[\begin{array}{c}
\dot{\mathrm{P}}_{1} \\
\dot{\mathrm{P}}_{2} \\
\dot{\lambda}
\end{array}\right]=\left[\begin{array}{ccc}
\mathrm{C}_{1} & 0 & \mathrm{~S}_{3} \\
0 & \mathrm{C}_{2} & \mathrm{~S}_{4} \\
\mathrm{~S}_{1} & \mathrm{~S}_{2} & 0
\end{array}\right]\left[\begin{array}{c}
\mathrm{P}_{1} \\
\mathrm{P}_{2} \\
\lambda
\end{array}\right]
$$

Where $P_{1}$ is the redundant power vector. Eliminating $\lambda$ and $P_{1}$ yields the following reduced differential equation. 


$$
\begin{aligned}
\dot{P}_{2} & =\left|T_{2}+S_{4} S_{3}^{-1} T_{1} S_{1}^{-1} S_{2}\right|^{-1}\left|C_{2}+S_{4} S_{3}^{-1} C_{1} s_{1}^{-1} S_{2}\right| P_{2} \\
& =\mathrm{HP}_{2}
\end{aligned}
$$

The stability of the deregulation system is investigated by the eigenvalues of the characteristic matrix $\mathrm{H}$.

\section{The modified dynamic equation:}

In order to research the desired strategic steady solution, we will modify the optimal dynamic equation. The modified procedure is described as the following.

Set $\Delta \mathrm{B}$ to be "provider's pricing difference matrix" that is dependent on $\Delta \lambda=\lambda-\lambda^{\circ}$. $\lambda^{0}$ and $\lambda$ are the optimal and desired prices, respectively.

Now we express the optimal dynamic and the modified dynamic equations as the following.

The optimal dynamic equation:

$$
\left(\begin{array}{ll}
T & 0 \\
0 & 0
\end{array}\right)\left(\begin{array}{l}
\dot{P}^{o} \\
\dot{\lambda}^{\circ}
\end{array}\right)=\left(\begin{array}{ll}
Q_{1} & Q_{2} \\
Q_{3} & Q_{4}
\end{array}\right)\left(\begin{array}{l}
P^{o} \\
\lambda^{o}
\end{array}\right)-\left(\begin{array}{l}
B \\
L
\end{array}\right)
$$

The modified dynamic equation:

$$
\left(\begin{array}{ll}
T & 0 \\
0 & 0
\end{array}\right)\left(\begin{array}{l}
\dot{P} \\
\dot{\lambda}
\end{array}\right)=\left(\begin{array}{cc}
Q_{1}+\Delta Q_{1} & Q_{2}+\Delta Q_{2} \\
Q_{3} & Q_{4}
\end{array}\right)\left(\begin{array}{l}
P \\
\lambda
\end{array}\right)-\left(\begin{array}{c}
B+\Delta B \\
L
\end{array}\right)
$$

where $\left(\Delta Q_{1}, \Delta Q_{2}\right)$ is the necessary terms that we must modify the dynamic equation to reach the strategic steady solution $\mathrm{S}=(\mathrm{P}, \lambda)^{\mathrm{T}}$. By some algebraic algebraic manipulations, we obtain the following.

$$
\left(\Delta Q_{1}, \Delta Q_{2}\right)=\left(\Delta B-Q_{2} \Delta \lambda\right)\left(S^{T} S\right)^{-1} S^{T}
$$

As long as the strategic steady solution $S$ is assigned. Then the $\left(\Delta Q_{1}, \Delta Q_{2}\right)$ can be obtained by Eq.(13).

\section{Simulation Results}

We use IEEE-30 bus shown in Figure 6 to demonstrate the proposed method and assign bus $13,10,5,19$ to providers $A, B, C, D$, respectively. The load is at bus 5 . Line 1 is the branch between bus 5 and bus 7. For easy comparison, we fixed the following parameters in Table 1.

Table 1: The parameters of simulation

\begin{tabular}{|l|c|c|c|c|c|c|c|c|}
\hline parameter & $\mathrm{T}_{\mathrm{A}}$ & $\mathrm{T}_{\mathrm{B}}$ & $\mathrm{T}_{\mathrm{C}}$ & $\mathrm{T}_{\mathrm{D}}$ & $\mathrm{b}_{\mathrm{A}}$ & $\mathrm{b}_{\mathrm{B}}$ & $\mathrm{b}_{\mathrm{C}}$ & $\mathrm{b}_{\mathrm{D}}$ \\
\hline value & 3 & 2 & 1 & 2 & 0.2 & 0.1 & 0.2 & 0.2 \\
\hline parameter & $\mathrm{C}_{\mathrm{A}}$ & $\mathrm{C}_{\mathrm{B}}$ & $\mathrm{C}_{\mathrm{C}}$ & $\mathrm{C}_{\mathrm{D}}$ & $\mathrm{E}_{\mathrm{L}}$ & $\mathrm{L}$ & $\alpha$ & $\beta$ \\
\hline value & 0.03 & 0.02 & 0.05 & 0.06 & 0.21 & 8 & 0.0502 & 0.0313 \\
\hline
\end{tabular}

\begin{tabular}{|c|c|c|c|c|c|c|c|}
\hline policy & $\begin{array}{l}\lambda_{1} \\
\lambda_{2} \\
\lambda_{1}\end{array}$ & $\begin{array}{l}P_{A} \\
P_{B} \\
P_{C} \\
P_{D}\end{array}$ & $f_{1}$ & $f_{2}$ & $f_{3}$ & Pool cost & $\begin{array}{l}\text { Eigen. } \\
\text { value }\end{array}$ \\
\hline uniform & $\begin{array}{c}5 \\
\text { NA } \\
\text { NA }\end{array}$ & $\begin{array}{l}2.8307 \\
2.1693 \\
0.7500 \\
2.2500\end{array}$ & 17.053 & 10.5826 & 10.6481 & 40 & \begin{tabular}{|l|}
0.0207 \\
-0.0021 \\
-0.0215
\end{tabular} \\
\hline UK_m & $\begin{array}{c}1 \\
5 \\
\text { NA }\end{array}$ & $\begin{array}{l}2.8307 \\
2.1693 \\
0.7500 \\
2.2500 \\
\end{array}$ & 5.7303 & 1.9053 & 10.6481 & 23 & $\begin{array}{l}-0.0181 \\
-0.0945\end{array}$ \\
\hline UK_I & $\begin{array}{c}1 \\
5 \\
\text { NA }\end{array}$ & $\begin{array}{l}0.9601 \\
5.1694 \\
0.7500 \\
1.1205\end{array}$ & 5.0902 & 4.3852 & 5.3407 & 17.3525 & $\begin{array}{c}0.0307 \\
-0.0801\end{array}$ \\
\hline Dis_m & $\begin{array}{c}0.75 \\
1 \\
5\end{array}$ & $\begin{array}{l}2.8307 \\
2.1693 \\
0.7500 \\
2.2500\end{array}$ & 5.0227 & 1.9053 & 10.6481 & 19.2923 & -0.1308 \\
\hline Dis_ I & $\begin{array}{c}0.75 \\
1 \\
5\end{array}$ & $\begin{array}{l}0.9601 \\
5.1694 \\
0.7500 \\
1.1205\end{array}$ & 4.1002 & 4.3852 & 5.3407 & 15.2420 & -0.1342 \\
\hline
\end{tabular}

The simulation results of the different policies are summarized in Table 2. According to Eq.(10), we set $\left(P_{C}+P_{D}\right)^{\text {merit-order }}=3,\left(P_{C}+P_{D}\right)^{\text {least }}=1.8705$.
Table 2: Simulation results of the different policies

The abbreviation of Table 2:

Uniform: uniform policy.

UK_m: U.K policy with merit-order curtailment

UK I: U.K policy with least curtailment

Dis_m: Discriminatory policy with merit-order curtailment

Dis_l: Discriminatory policy with least curtailment

The uniform policy has only one buying price level. The UK_m and UK_l have two levels of buying price. The Dis $m$ and Dis $I$ have three levels of buying price. $S=(P$, $\lambda)^{\bar{T}}$ in the first two columns of Table 2 denotes the strategic results. We notice the index of the eigenvalues. It shows that the uniform and UK_I policies are unstable. The instability means that such transaction is impractical. Its solution will diverse as time increasing.

Next we can use the derived dynamic equations to portray the stable and unstable region within the load $L=8$ range. The feasible region denotes that all of the providers do not supply negative power of steady solution. For the discriminatory policy, the two curtailment methods have the same dynamic equations as mentioned last section. Therefore, we only portray one discriminatory drawing. The drawings are shown in figure 2 to figure 5 . The data supports the participant to decide which strategy is practical.

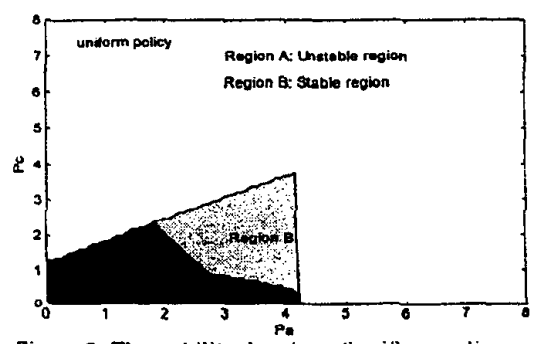

Figure 2: The stability drawing of uniform policy 


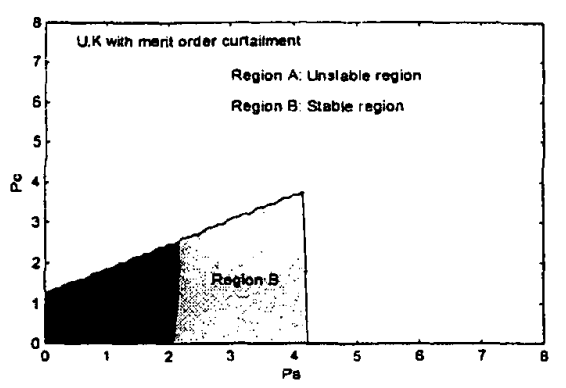

Figure 3: The stability drawing of UK_m policy

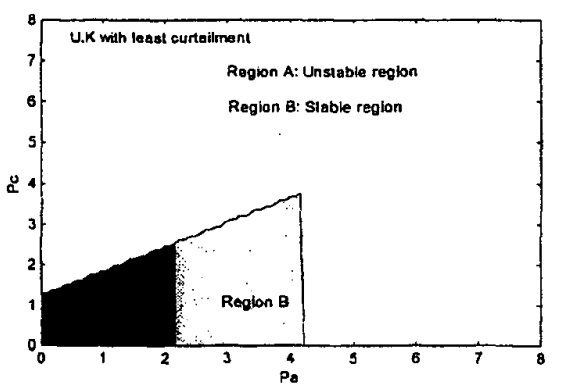

Figure 4: The stability drawing of UK_I policy

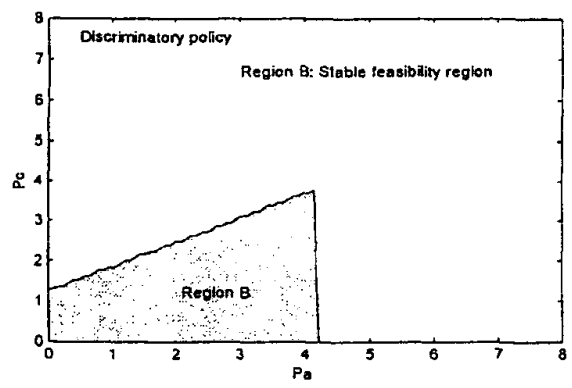

Figure 5: The stability drawing of discriminatory policy

\section{Conclusions}

Accompany with the progress of power deregulation, the power dispatch center has changed its role from ordering to supervising. The ISO needs to inspect the security of transaction and adopts the necessary curtailment. The paper studies the stability of power transaction. The unstable transaction means that the strategy is impractical. Although there have been several papers dealing with the topic, they have not considered the strategic behavior yet. We successfully use IEEE-30 bus to investigate the stability of distinct policies. It shows that the uniform policy is most unstable. Besides that, the unstable region is available for the congestion management of ISO and the references of participants..

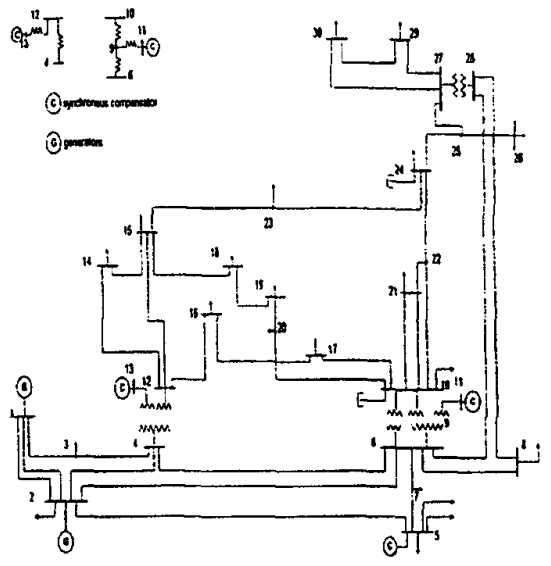

References:

Figure 6 IEEE 30-bus system

1. J.P.Aubin, "Mathematical methods of game and economic theory" Amsterdam, North-Holland Publishing company, 1982.

2. R.W.Ferrero, S.M.Shahidepour, V.C.Ramesh, "Transaction analysis in deregulated power systems using game theory" IEEE Trans. on power system, 1997, 12(3), $1340-1347$

3. X.Bai, S.M.Shahidepour, V.C.Ramesh, E.Yu, "Transmission analysis by nash game method "IEEE Trans. on power system, 1997, 12(3), 1046-1052.

4. C.W.Richter, Jr, G.B.Sheble" Genetic algorithm evoluation of utility bidding strategies for the competitive marketplace" IEEE Trans. on power system, 1998, 13(1), 256-261.

5. J.W.Lamont, S.Rajan, "Strategic bidding in an energy brokerage" IEEE Trans, on power system, 1997,12(4), 1729-1733.

6. K.W.Doty, P.L.McEntire “ An analysis of electric power brokerage systems" IEEE Trans. on power apparatus and system, 1982, PAS-101, 389-396.

7. G.Fahd, D.A.Richards, G.B.Sheble "The implementation of an energy brokerage system using linear programming" IEEE Trans. on power apparatus and system, 1992, 7, 90-96.

8.G.Fahd, G.B.Sheble "Optimal power flow emulation of interchange brokerage system using linear programming" IEEE Trans. on power apparatus and system, 1992, 7,497-504.

9.R.Baldick, R.J.Kaye, F.F.Wu, "Electricity tariffs under imperfect knowiedge of participant benefits" IEEE Trans on power system, 1992, 7(4), 1471-1480.

10.F.F.Wu "Coordinated multilateral trades for electric power networks" Power System Computation conference, 1996, 786-792.

11.B.R.Szkuta, L.A.Sanabria, T.S.Dillon "Electricity price short term forecasting using artificial neural networks" IEEE Trans. on power system, 1999, 14(3), 851-0857.

12.F.Alvarodo "The dynamic of power system market" Technical report PS ERC-97-01, Power System Engineering Research Consortium, the university of Wasconsin, March, 1997.

13.P.A.Samuelson "Foundations of economic analysis" Enlarged edition, Harvard university press, 1983.

14.F.Alvarado "The stability of power system markets" IEEE Power Engineering Society, PE-450-PWRS-0-05-1998.

15.L.L.Freris, A.M.Sasson "Investigation of the load flow problem" IEE proc., 1968, $115(10), 1459-1470$.

16.K.Seeley, J.Lawarree, C.C.Liu "Analysis of electricity inarket rules and their effects on strategic behavior in a non-congestive grid" PE-226-PWRS-0-01-1999

\section{Author Biographies:}

Chong-Yih Li was born in Taiwan in 1963. He received the B.S. degree in electronic engineering from Chung-Yuan Christian University in 1986, M.S. degree in electrical engineering from National Tsing-Hua University in 1988. He is electrical engineer in Taiwan Power Company and doctoral student in electrical engineering of National Taiwan University, now.

Chih-Wen Liu was born in Taiwan in 1964. He received the B.S. degree in electrical engineering from National Taiwan University in 1987, Ph.D. degree in electrical engineering from Comell University in 1994. Since 1994, he has been with National Taiwan University, where he is associate professor of electrical engineering. His research interests lie in power system computer application. 